\title{
ENT Manifestations in COVID-19 Positive Patients
}

\author{
Dr. Vaibhav Kuchhal \\ GMC Haldwani
}

Dr. Shahzad Ahmad

GMC Haldwani

Dr. Priyanka Chaurasia ( $\nabla$ chourasiapriyanka38@gmail.com )

GMC Haldwani

Dr. Pradeep Rawat

GMC Haldwani https://orcid.org/0000-0003-1340-2140

\section{Research Article}

Keywords: CoViD-19, loss of taste, loss of smell, ENT manifestations

Posted Date: October 15th, 2020

DOI: https://doi.org/10.21203/rs.3.rs-90203/v1

License: () (i) This work is licensed under a Creative Commons Attribution 4.0 International License. Read Full License 


\section{Abstract}

AIM: To study the status of ear, nose and throat in the patients diagnosed with the CoViD-19.

MATERIAL AND METHODS: This is a perspective study in patients admitted in the isolation wards of Dr. Sushila Tiwari Hospital Haldwani between March 2020 and July 2020. There were 465 symptomatic patients out of total 727 patients diagnosed with CoViD19 .

RESULT: Since the CoViD-19 pandemic has begun, many individuals have noted many ENT manifestations along with the other general symptoms like fever(395 pt)>cough (326 pt) >weakness/fatigue (190 pt) >difficulty in breathing (89 pt) >muscle ache (58 pt ) >headache (56 pt) \& diarrhoea (24 pt). Among ENT manifestations throat symptoms were found most common including loss of taste in 74 pt. It was noticed that most commonly sweet and salt goes first then sour taste vanish and bitter and chilli taste goes in the end, most of them were reversible after 2-3 weeks. Among nasal symptoms nasal congestion was found most common (72 pt) followed by loss of smell ( $88 \mathrm{pt})$, nasal blockage ( $66 \mathrm{pt})$ \& runny nose (47 pt). loss of smell was also reversible in most of the cases after 2-3 weeks. Ear symptoms were not so commonly found. Although itching in bilateral ear, sensation of ear fullness, ear ache and conductive hearing loss were complained by some patients.

CONCLUSION: This study represents the initial findings of all individuals with ENT manifestations along with the chemosensory loss in the symptomatic confirmed covid-19 patients. During the ongoing COVID-19 pandemic, changes in smell or taste are not being considered indicative of possible COVID-19 infection-especially in the absence of other high-suspicion symptoms.

\section{Introduction}

Coronaviruses are a group of related RNA viruses that cause diseases in mammals and birds. In humans, these viruses cause respiratory tract infections that can range from mild to lethal. Mild illnesses include some cases of the common cold (which is also caused by other viruses, predominantly rhinoviruses), while more lethal varieties can cause SARS, MERS, and COVID-19. They are enveloped viruses with a positive-sense single-stranded RNA genome and a nucleocapsid of helical symmetry.[7] The genome size of coronaviruses ranges from approximately 26 to 32 kilobases, one of the largest among RNA viruses. [8] They have characteristic club-shaped spikes that project from their surface, which in electron micrographs create an image reminiscent of the solar corona, from which their name derives.[9] The coronavirus surface spikes are homotrimers of the S protein, which is composed of an S1 and $\mathrm{S} 2$ subunit. The homotrimeric $\mathrm{S}$ protein is a class I fusion protein which mediates the receptor binding and membrane fusion between the virus and host cell. The S1 subunit forms the head of the spike and has the receptor binding domain (RBD). The S2 subunit forms the stem which anchors the spike in the viral envelope and on protease activation enables fusion. The $\mathrm{E}$ and $\mathrm{M}$ protein are important in forming the viral envelope and maintaining its structural shape. Transmission Infected carriers are able to shed viruses into the environment. The interaction of the coronavirus spike protein with its complementary cell receptor is central in determining the tissue tropism, infectivity, and species range of the released virus. Coronaviruses mainly target epithelial cells.[5] They are transmitted from one host to another host, depending on the coronavirus species, by either an aerosol, fomite, or fecal-oral route. The incubation time for COVID-19 since the exposure to SARS-CoV-2 is believed to reach 14 days, nevertheless, the majority of patients develop COVID-19 disease after 4-5 days (range between 2 and 7 days) after being infected [4, 5]. COVID-19 remains contagious even during the latency period, thus patients before clinical COVID-19 presentation can transfer the virus to others.

Infection in humans

In December 2019, a pneumonia outbreak was reported in Wuhan, China. On 31 December 2019, the outbreak was traced to a novel strain of coronavirus, which was given the interim name 2019-nCoV by the World Health Organization (WHO), later renamed SARSCoV-2 by the International Committee on Taxonomy of Viruses. As of 31 july 2020, there have been at least 675,545 confirmed deaths and more than 17,406,644 confirmed cases in the COVID-19 pandemic world wide and at least 35,745 deaths and more than $1,638,827$ confirmed cases of COVID-19 IN India.

\section{$\underline{\text { AlM }}$}

The aim of study is to see ENT manifestations in corona affected patients admitted in the isolation wards of Dr. Sushila Tiwari Hospital haldwani since 1/4/2020 till 31/7/2020 . 
Sampling technique: Total enumeration.

Prevalance of symptomatic patients admitted in STH: 0.64 (64\%)

Inclusion criteria : All symptomatic covid-19 affected patients admitted in isolation wards of STH haldwani.

Exclusion criteria : patients or guardian refusal for consent.

\section{Result}

From 01/04/2020 till 31/07/2020, a total of 465 respondents met inclusion. Which were COVID-19 positive symptomatic patients by testing or diagnosis by a health care practitioner in STH Haldwani. Although most respondents experienced other major COVID-19related symptoms during or after their chemosensory change.

Table 1. Coexisting Symptoms Associated With Chemosensory Changes.

Respondents, No. (\%)

Symptoms

Symptomatic COVID-19 patients $(\underline{n}=465)$

CDC major symptoms

Difficulty breathing

$89(19.2 \%)$

Cough

$326(70.2 \%)$

Fever

$395(85 \%)$

Muscle aches

$58(12.5 \%)$

Headaches

$56(12 \%)$

Other symptoms

Weakness or fatigue

$190(41 \%)$

Diarrhea

24(5.2\%)

Among ENT manifestations throat symptoms were found most common including loss of taste in 74 pt. It was noticed that most commonly sweet and salt goes first then sour taste vanish and bitter and chilli taste goes in the end, most of them were reversible after 2-3 weeks. Among nasal symptoms nasal congestion was found most common (72 pt) followed by loss of smell (88 pt), nasal blockage (66 pt) \& runny nose (47 pt). loss of smell was also reversible in most of the cases after 2-3 weeks.

Ear symptoms were not so commonly found. Although itching in bilateral ear, sensation of ear fullness, ear ache and conductive hearing loss were complained by some patients.

\section{Discussion}


To date, preliminary work has demonstrated a high incidence of otorhinolaryngological changes along with smell or taste change in patients with COVID-19. However, the wide variation in populations studied, methods of recruitment, Table 1. Coexisting symptoms at the time of ENT manifestations. Table 2. Shows patients presented with various nasal symptoms.Figure 2. Subjective smell prior to and while symptomatic (all patients) presented with partial (50) and complete (38) loss of smell which is completely reversible in most patients.

Table 3. Ear manifestations prior to and while symptomatic (all patients). Table 4. Throat symptoms prior to and while symptomatic (all patients) Figure 2. Shows the pattern of loss of taste sensations which was completely reversible in most of the cases. Irrespective, chemosensory changes associated with COVID-19 are well established, leading most health organizations worldwide to include acute changes in smell or taste among symptoms suggestive of COVID-19. Despite this, many patients with new-onset chemosensory deficits are still not suspected of COVID-19 infection or tested as such. Reasons for lack of testing are likely several and may owe largely to limited availability as well as a lack of understanding in the lay and medical communities of the association between smell/taste and COVID-19. ${ }^{1}$ Among all respondents experiencing olfactory disturbances in our study, $11.7 \%$ cited chemosensory disturbances as their first or only symptom of disease. This is consistent with $11.7 \%$ of hospitalized patients with COVID-19 reporting smell or taste disturbances as the initial presenting symptom but substantially higher than other reports based on patient populations with presumably milder disease. The co-occurrence of multiple well known symptoms of COVID-19 suggests that many of these respondents were undiagnosed COVID-19 infections. Some confirmed covid-19 Patients also presented with subjective discomfort in the bilateral ears and bilateral mild conductive hearing loss.

\section{Conclusion}

This study represents the initial findings of a longitudinal survey of all individuals with ENT manifestations along with the chemosensory loss in the symptomatic confirmed covid-19 patients admitted in the isolation wards of Dr. Sushila Tiwari hospital Haldwani during the COVID-19 pandemic. Patients may present with smell or taste loss before other symptoms and experience complete subjective loss of smell or taste. During the ongoing COVID-19 pandemic, changes in smell or taste are not being considered indicative of possible COVID-19 infection- especially in the absence of other high-suspicion symptoms.

\section{Declarations}

FUNDING: This research received no specific grant from any funding agency.

CONFLICT OF INTEREST: The author hereby declare that there is no potential conflicts of interest with respect to research and publication of this article.

ETHICAL STANDARDS: As it is a study involving human participants, ethical clearance was obtained from the institution.

INFORMED CONSENT: Informed consent was obtained for all participants included in the study.

\section{References}

1. Coelho DH, Kons ZA, Costanzo RM, Reiter ER. Subjective Changes in Smell and Taste During the COVID-19 Pandemic: A National Survey-Preliminary Results. Otolaryngol Head Neck Surg. 2020 Aug;163(2):302-306. doi: 10.1177/0194599820929957. Epub 2020 May 19. PMID: 32423359.

2. Korea Times. 15\% of COVID-19 patients lose sense of smell or taste: data. Accessed April 25, 2020. https://www.koreatimes.co.kr/www/nation/2020/03/119_286790.html

3. Bagheri SHR, Asghari AM, Farhadi M, et al. Coincidence of COVID-19 epidemic and olfactory dysfunction outbreak. medRxiv. Published online March 27, 2020. doi:10.1101/2020 .03.23.20041889

4. Schmitt P-P. Virologe Hendrik Streeck: „Wir haben neue Symptome entdeckt.” FAZ.NET. Accessed April 25, 2020. https://www.faz.net/1.6681450 
5. Tong JY, Wong A, Zhu D, Fastenberg JH, Tham T. The Prevalence of Olfactory and Gustatory Dysfunction in COVID-19 Patients: A Systematic Review and Meta-analysis. Otolaryngol Head Neck Surg. 2020 Jul;163(1):3-11. doi: 10.1177/0194599820926473. Epub 2020 May 5. PMID: 32369429.

6. Moein ST, Hashemian SMR, Mansourafshar B, Khorram-Tousi A, Tabarsi P, Doty RL. Smell dysfunction: a biomarker for COVID19. Int Forum Allergy Rhinol. Published online April 18, 2020. doi:10.1002/alr.22587

7. Villalba NL, Maouche Y, Ortiz MBA, et al. Anosmia and dysgeusia in the absence of other respiratory diseases: should COVID-19 infection be considered? Eur J Case Rep Intern Med. 2020;7(4):001641.

8. Heidari F, Karimi E, Firouzifar M, et al. Anosmia as a prominent symptom of COVID-19 infection. Rhinology. Published online April 22, 2020. doi:10.4193/Rhin20.140

9. Centers for Disease Control and Prevention. Coronavirus disease 2019 (COVID-19)-symptoms. Published April 17, 2020. Accessed April 25, 2020. https://www.cdc.gov/coronavirus/ 2019-ncov/symptoms-testing/symptoms.html

10. Harris PA, Taylor R, Thielke R, Payne J, Gonzalez N, Conde JG. Research electronic data capture (REDCap)-a metadatadriven methodology and workflow process for providing translational research informatics support. J Biomed Inform. 2009; 42(2):377381.

11. Harris PA, Taylor R, Minor BL, et al. The REDCap consortium: building an international community of software platform partners. J Biomed Inform. 2019;95:103208.

12. Beltra'n-Corbellini A' , Chico-Garcl'a JL, Martı́nez-Poles J,et al. Acute-onset smell and taste disorders in the context of COVID19: a pilot multicenter PCR-based case-control study. Eur J Neurol. Published online April 22, 2020. doi:10.1111/ene.14273

13. Yan $\mathrm{CH}$, Faraji F, Prajapati DP, Boone CE, DeConde AS. Association of chemosensory dysfunction and Covid-19 in patients presenting with influenza-like symptoms. Int Forum Allergy Rhinol. Published online April 12, 2020. doi:10.1002/ alr.22579

14. Lechien JR, Chiesa-Estomba CM, De Siati DR, et al. Olfactory and gustatory dysfunctions as a clinical presentation of mild-tomoderate forms of the coronavirus disease (COVIDCoelho et al 305 19): a multicenter European study. Eur Arch Otorhinolaryngol. Published online April 6, 2020. doi:10.1007/s00405-020- 05965-1

15. Hopkins C, Surda P, Kumar N. Presentation of new onset anosmia during the COVID-19 pandemic. Rhinology. Published online April 11, 2020. doi:10.4193/Rhin20.116

16. Kaye R, Chang CD, Kazahaya K, Brereton J, Denneny J. COVID-19 anosmia reporting tool: initial findings. Otolaryngol Head Neck Surg. Published online April 28, 2020. doi:10.1177/ 0194599820922992

\section{Tables}

Due to technical limitations, the tables can be found in the supplementary section.

\section{Figures}






\section{Figure 1}

Shows grading and reversibility found in respondents.

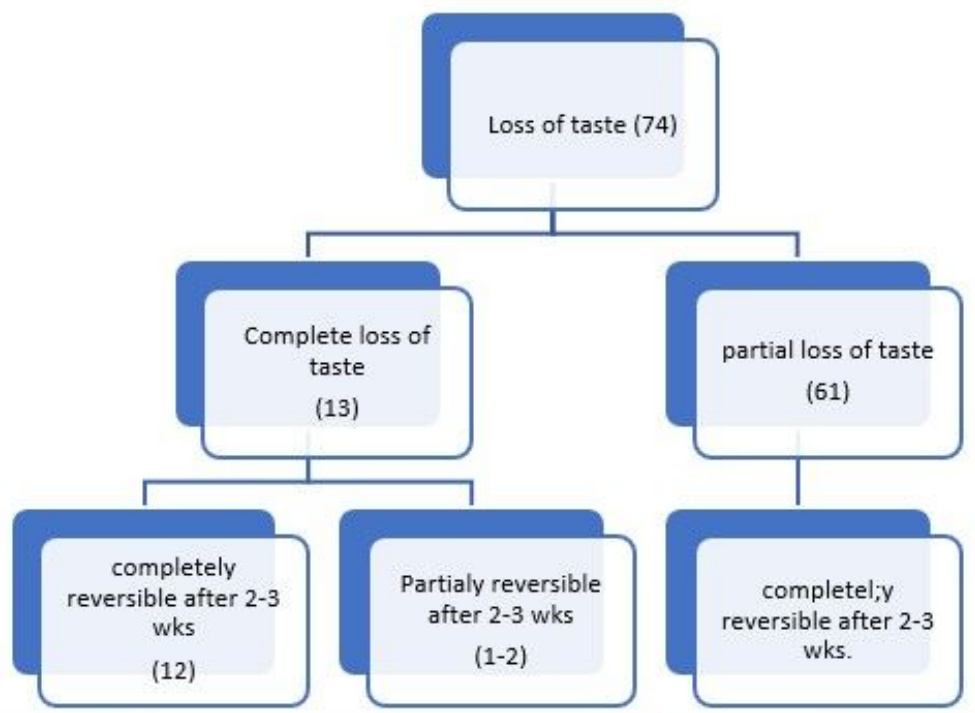

most commonly

sweet and salt goes first then sour taste

vanish.
Bitter and chilli taste goes in the end 
Figure 2

Shows a brief description about grading, reversibility and type of loss of taste found.

\section{Supplementary Files}

This is a list of supplementary files associated with this preprint. Click to download.

- Tablesforpaper.docx 